\title{
Simulation of unsteady aerodynamic loads on high-speed trains in fluctuating crosswinds
}

\author{
Mengge Yu $\cdot$ Jiye Zhang $\cdot$ Weihua Zhang
}

Received: 28 February 2013/Revised: 17 April 2013/Accepted: 20 April 2013/Published online: 6 June 2013

(C) The Author(s) 2013. This article is published with open access at Springerlink.com

\begin{abstract}
To study the unsteady aerodynamic loads of high-speed trains in fluctuating crosswinds, the fluctuating winds of a moving point shifting with high-speed trains are calculated in this paper based on Cooper theory and harmonic superposition method. The computational fluid dynamics method is used to obtain the aerodynamic load coefficients at different mean yaw angles, and the aerodynamic admittance function is introduced to calculate unsteady aerodynamic loads of high-speed trains in fluctuating winds. Using this method, the standard deviation and maximum value of the aerodynamic force (moment) are simulated. The results show that when the train speed is fixed, the varying mean wind speeds have large impact on the fluctuating value of the wind speeds and aerodynamic loads; in contrast, when the wind speed is fixed, the varying train speeds have little impact on the fluctuating value of the wind speeds or aerodynamic loads. The ratio of standard deviation to $0.5 \rho K \bar{u}^{2}$, or maximum value to $0.5 \rho K \bar{u}^{2}$, can be expressed as the function of mean yaw angle. The peak factors of the side force and roll moment are the same ( $\sim 3.28$ ), the peak factor of the lift force is $\sim 3.33$, and the peak factors of the yaw moment and pitch moment are also the same $(\sim 3.77)$.
\end{abstract}

Keywords Fluctuating winds - Unsteady aerodynamic loads $\cdot$ Yaw angle $\cdot$ Peak factor

\footnotetext{
M. Yu ( $\) \cdot$ J. Zhang $\cdot$ W. Zhang

Traction Power State Key Laboratory, Southwest Jiaotong

University, Chengdu 610031, China

e-mail: yumengge0627@163.com

W. Zhang

e-mail: yzhang@home.swjtu.edu.cn
}

\section{Introduction}

As a result of the increasing of the train speed, the aerodynamics of high-speed trains has attracted great attention. Strong crosswinds seriously affect the operation safety of high-speed trains. Incidents of train derailments and overturns in strong winds have been reported from around the world with recent incidents being reported in Japan, Italy, Belgium, Switzerland, and China. A great deal of research has been carried out to investigate the operational safety of high-speed trains. In the past, most research on the aerodynamic performance and safety characteristics of high-speed trains under crosswinds are based on uniform wind hypothesis [1-4]. However, the natural winds are not constant but instantaneous, and have fluctuating characteristics. The analysis based on uniform wind hypothesis is much different from the actual situation; thus, it is important to carry out the research on the aerodynamic characteristics of high-speed trains in fluctuating winds. At present, extensive research work has been carried out on the vehicle-bridge coupling vibration $[5,6]$, in which the fluctuating winds at fixed points are considered, and the unsteady aerodynamic loads of highspeed trains are calculated based on the quasi-steady expression. Cooper [7] derived the wind spectrum of fluctuating winds at a moving point shifting with highspeed trains based on von Karman spectrum, which is Cooper theory. Baker [8] found that, compared with the spectrum of fluctuating winds at a fixed point, the wind spectrum of a moving point based on the Cooper theory will move to the higher frequency part. The quasi-steady expression assumes that force fluctuations follow velocity fluctuations. In reality, the quasi-steady assumption does not hold completely, and the force fluctuations do not completely follow the velocity fluctuations as the 
small-scale turbulence in the oncoming wind is not fully correlated over the entire exposed area of a train. The aerodynamic admittance function can solve the problem. This parameter is a normalized ratio of the force spectrum to the wind spectrum and can be measured in experiments. Baker et al. $[9,10]$ carried out several wind tunnel tests, and obtained the aerodynamic admittance function of high-speed trains. Recently, Baker [11] has studied the variation of the unsteady side force and lift force of a vehicle at different operating speeds according to the actual situation of trains. However, the characteristics of aerodynamic moments and the impact of yaw angle on statistical characteristics of aerodynamic loads are not considered in Baker's research.

In this paper, the fluctuating winds of a moving point shifting with high-speed trains are simulated based on Cooper theory and harmonic superposition method. The aerodynamic admittance function is introduced to compute unsteady aerodynamic loads of high-speed trains at different train speeds $(200-400 \mathrm{~km} / \mathrm{h})$ and different mean wind speeds $(10-30 \mathrm{~m} / \mathrm{s})$, and statistical characteristics of aerodynamic loads at different yaw angles are analyzed.

\section{Numerical simulation of fluctuating crosswinds}

Wind observation records show that the instantaneous wind consists of two parts: a mean wind with a period of $10 \mathrm{~min}$, and a fluctuating wind with a period of several seconds. The instantaneous wind speed can be expressed as

$w=\bar{w}+w^{\prime}$,

where $\bar{w}$ is the mean wind speed, and $w^{\prime}$ is the fluctuating value of wind speed.

We assume that the train is traveling along a straight track, with the mean wind direction normal to the track. The velocity vector diagram is shown in Fig. $1 . \alpha$ is the

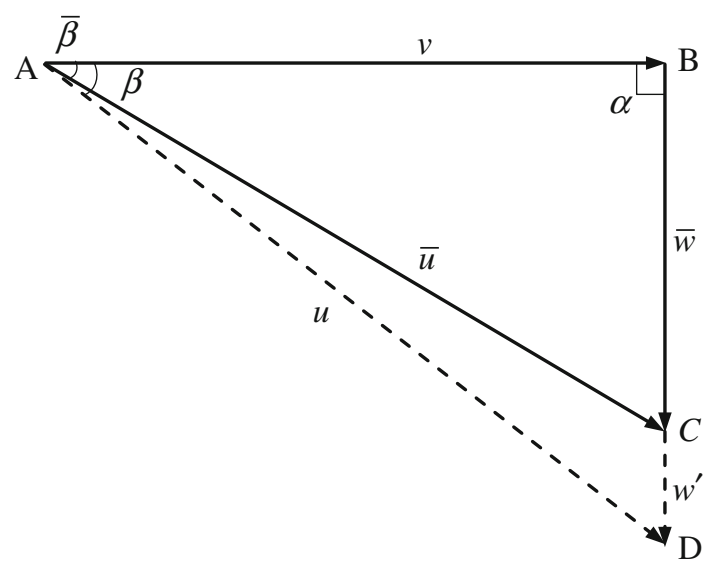

Fig. 1 Velocity vector diagram mean wind direction, and $\alpha=90^{\circ} ; \bar{\beta}$ is the angle between the mean synthetic wind $\bar{u}$ and the track, which is called the mean yaw angle in the paper; $\beta$ is the angle between the synthetic wind $u$ and the track, which is called yaw angle; and $v$ is the train speed.

In this paper, we simulate one fluctuating wind time series at the location of the train at any instance. This is done by a simple decomposition of the wind spectrum relative to the moving train into series of sinusoidal velocity variations of random phase, and a combination of these time series into the fluctuating wind time series at the train position. The power spectral density adopted is that of Cooper [7], which is given by

$$
\begin{aligned}
\frac{n S_{w}}{\sigma_{w}^{2}}= & {\left[\frac{4\left(n L^{\prime} / \bar{u}\right)}{\left(1+70.8\left(n L^{\prime} / \bar{u}\right)^{2}\right)^{5 / 6}}\right] } \\
& \times\left[\left(\frac{\bar{w}}{\bar{u}}\right)^{2}+\left(1-\left(\frac{\bar{w}}{\bar{u}}\right)^{2}\right) \frac{0.5+94.4\left(n L^{\prime} / \bar{u}\right)^{2}}{1+70.8\left(\left(n L^{\prime} / \bar{u}\right)^{2}\right)}\right]
\end{aligned}
$$

where

$L^{\prime}=L_{w}^{x}\left(\left(\frac{\bar{w}}{\bar{u}}\right)^{2}+4\left(\frac{L_{w}^{y}}{L_{w}^{x}}\right)^{2}\left(1-\left(\frac{\bar{w}}{\bar{u}}\right)^{2}\right)\right)^{0.5} ;$

$n S_{w} / \sigma_{w}^{2}$ is the dimensionless power spectral density of the fluctuating wind speed, $n$ is the frequency, $S_{w}$ is the power spectral density of the fluctuating wind speed, and $\sigma_{w}$ is the variance of the fluctuating wind speed: $\sigma_{w}=I_{z} \bar{w} ; I_{z}$ is the turbulence intensity; $L_{w}^{x}$ is the longitudinal turbulence scale; and $L_{w}^{y}$ is the lateral turbulence scale.

Turbulence intensity can be calculated as follows [12]:

$I_{z}=\frac{1-5 \times 10^{-5}\left(\log \left(z_{0} / 0.05\right)+2\right)^{7}}{\ln \left(z / z_{0}\right)}$,

where $z_{0}$ is the roughness height, and $z$ is the height above ground.

The longitudinal and lateral turbulence scales can be calculated, respectively, as [13]

$L_{w}^{x}=50 \times z^{0.35} / z_{0}^{0.063}$,

$L_{w}^{y}=0.42 L_{w}^{x}$.

The values of the spectral density at discrete frequencies $n_{j}$ are then used to calculate the fluctuating wind time series at the train position:

$w^{\prime}(t)=\sum_{j}\left[2 S_{w}\left(n_{j}\right) \Delta n_{j}\right]^{0.5} \sin \left(2 \pi n_{j} t+2 \pi r_{j}\right)$,

where $t$ is the time, $\Delta n_{j}$ is the frequency step, and $r_{j}$ is a random number between 0 and 1 . 


\section{Computational method of unsteady aerodynamic loads}

The unsteady aerodynamic force (moment) $F$ consists of a mean value $\bar{F}$ and a fluctuating value $F^{\prime}$. The mean value is

$\bar{F}=0.5 \rho K \bar{C}_{F} \bar{u}^{2}$,

where $\rho$ is the density of air; $K$ is $A$ for the aerodynamic force and $A H$ for the aerodynamic moment: $A$ is a reference area, and $H$ is a reference height; $\bar{C}_{F}$ is a load coefficient at the mean yaw angle $\bar{\beta}$, and $\bar{C}_{F} \equiv C_{F}(\bar{\beta})$.

Baker [11] obtained the formula of the unsteady aerodynamic forces of a moving train in fluctuating crosswinds. When aerodynamic force fluctuations follow wind fluctuations, the unsteady aerodynamic load can be expressed as

$F^{\prime}=\rho K \bar{C}_{F} \bar{w}\left(1+0.5 \bar{C}_{F}^{\prime} \cot \bar{\beta} / \bar{C}_{F}\right) w^{\prime}$,

where $\bar{C}_{F}^{\prime}$ is the derivative function of $C_{F}(\beta)$ at the mean yaw angle: $\bar{C}_{F}^{\prime} \equiv C_{F}^{\prime}(\bar{\beta})$.

Equation (9) is based on the quasi-steady expression which assumes that force fluctuations follow velocity fluctuations. By forming the autocorrelations and taking the Fourier transform of Eq. (9), we can derive the formula of the force spectrum and wind speed spectrum for the moving vehicle:

$S_{F}=\left(\rho K \bar{C}_{F} \bar{w}\left(1+0.5 \bar{C}_{F}^{\prime} \cot \bar{\beta} / \bar{C}_{F}\right)\right)^{2} S_{w}$,

where $S_{F}$ is the spectrum of force, and $S_{w}$ is the spectrum of wind speed.

However, in reality, the quasi-steady assumption does not hold completely, and the force fluctuations do not completely follow the velocity fluctuations as the smallscale turbulence in the oncoming wind is not fully correlated over the entire exposed area of a train. To allow for this, we introduce the aerodynamic admittance function $\chi^{2}$. Then, Eq. (10) can be modified to

$S_{F}=\left(\rho K \bar{C}_{F} \bar{w}\left(1+0.5 \bar{C}_{F}^{\prime} \cot \bar{\beta} / \bar{C}_{F}\right)\right)^{2} \cdot \chi^{2} S_{w}$.

From the above analysis, $w^{\prime}$ in Eq. (9) should be changed to $\tilde{w}^{\prime}$, i.e.,

$F^{\prime}=\rho K \bar{C}_{F} \bar{w}\left(1+0.5 \bar{C}_{F}^{\prime} \cot \bar{\beta} / \bar{C}_{F}\right) \tilde{w}^{\prime}$.

The spectral density of $\tilde{w}^{\prime}$ is $\chi^{2} S_{w}$, and the fluctuating wind time series $\tilde{w}^{\prime}$ can be calculated as follows:

$\tilde{w}^{\prime}(t)=\sum_{j}\left[2 \chi^{2}\left(n_{j}\right) S_{w}\left(n_{j}\right) \Delta n_{j}\right]^{0.5} \sin \left(2 \pi n_{j} t+2 \pi r_{j}\right)$.

Sterling et al. [14] assembled a significant amount of experimental data for aerodynamic admittances from a variety of full-scale wind tunnel tests on trains, and aerodynamic admittance of trains can be expressed as $\chi^{2}=\frac{1}{\left(1+\left(\bar{n} / \bar{n}^{\prime}\right)^{2}\right)^{2}}$,

$\bar{n}^{\prime}=\gamma \sin \bar{\beta}$,

where $\bar{n}$ is dimensionless frequency, $\gamma$ is 2.0 for the side force and 2.5 for the lift force. The aerodynamic admittance function of roll moment is the same as that of side force. The yaw moment and pitch moment fluctuations are considered to follow the wind speed fluctuations [13].

The aerodynamic admittances of side force and lift force when the mean yaw angle is $0.3 \mathrm{rad}$ are shown in Fig. 2 . The aerodynamic admittance functions of the side force and lift force have similar characteristics, and the latter is greater than the former. For a mean yaw angle of $0.3 \mathrm{rad}$, the aerodynamic admittance function is close to 1 when the dimensionless frequency $\bar{n}<0.1$. When $\bar{n}>0.1$, the aerodynamic admittance function continuously falls off until $\bar{n}>1$, and then reaches stability at 0 .

Figure 3 shows a plot of simulated aerodynamic admittance of side force at different mean yaw angles. The admittance function of the side force is moving to the

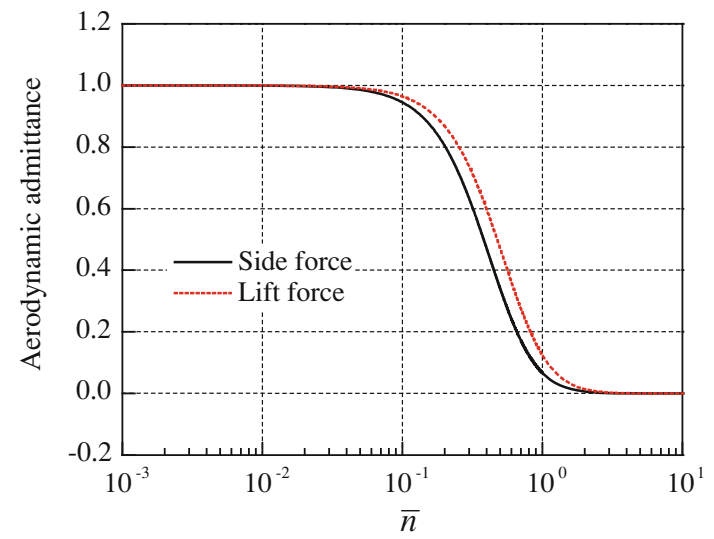

Fig. 2 Admittances of side force and lift force

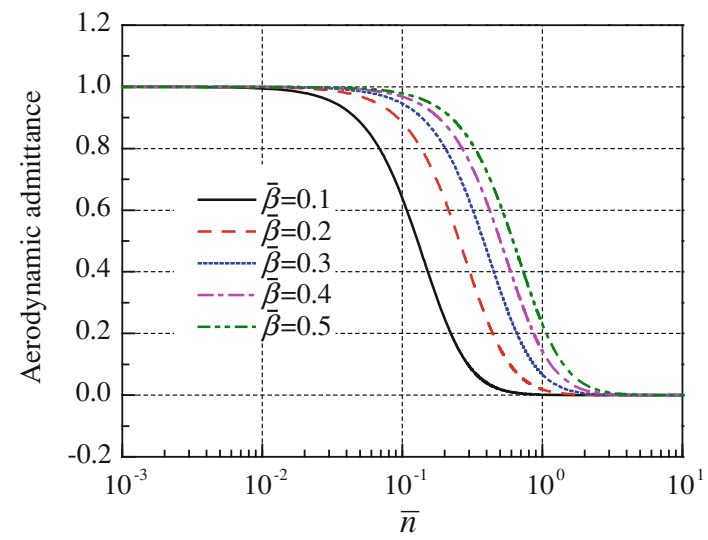

Fig. 3 Admittances of side force at different mean yaw angles 
higher part of the frequency with the increase of the mean yaw angles.

Computational fluid dynamics method is used to determine the mean aerodynamic coefficients. According to $\mathrm{Yu}$ et al. [15], the mean aerodynamic coefficients at different mean yaw angles are obtained. As the operation safety of the first car is the worst, only the mean aerodynamic coefficient of the first car is calculated. The mean aerodynamic coefficients depending on mean yaw angle are approximated by

$$
\begin{aligned}
& \bar{C}_{F s}=-0.01294+4.78604 \bar{\beta}+3.92245 \bar{\beta}^{2}, \\
& \bar{C}_{F l}=0.05141-4.89518 \bar{\beta}+23.441916 \bar{\beta}^{2}-17.60013 \bar{\beta}^{3},
\end{aligned}
$$

$\bar{C}_{M r}=0.01450-0.85537 \bar{\beta}-0.03651 \bar{\beta}^{2}$,

$\bar{C}_{M y}=-0.08886+11.54886 \bar{\beta}-8.47128 \bar{\beta}^{2}$,

$\bar{C}_{M p}=1.07723-7.60173 \bar{\beta}+58.10532 \bar{\beta}^{2}-79.91542 \bar{\beta}^{3}$

where $\bar{C}_{F s}$ is mean side force coefficient, $\bar{C}_{F l}$ is the mean lift force coefficient, $\bar{C}_{M r}$ is the mean roll moment coefficient, $\bar{C}_{M y}$ is the mean yaw moment coefficient, and $\bar{C}_{M p}$ is the mean pitch moment coefficient.

The correlation coefficients of the fitting formulas (16)(20) are 0.9999, 0.9996, 0.9998, 0.9994, and 0.9992, respectively.

\section{Result analysis}

In simulations, the train speeds are $200,250,300,350$, and $400 \mathrm{~km} / \mathrm{h}$. The mean wind speeds are 10, 15, 20, 25, and $30 \mathrm{~m} / \mathrm{s}$. The simulation time of fluctuating winds is $600 \mathrm{~s}$, and the fluctuating winds are generated every $0.05 \mathrm{~s}$.

\subsection{Simulation of the unsteady aerodynamic loads}

In order to verify the accuracy of the simulation, we compare the target spectrum with the simulated spectrum corresponding to the simulated wind time history with a train speed of $300 \mathrm{~km} / \mathrm{h}$ and a mean wind speed of $25 \mathrm{~m} / \mathrm{s}$, as shown in Fig. 4. The result indicates that the simulated spectrum of the simulated wind time history is in good agreement with the target spectrum, which verifies that the numerical simulation of fluctuating winds in the paper is reliable.

The time series of fluctuating value of wind speed are shown in Fig. 5. The time series of fluctuating values of side force corresponding to Fig. 5 are shown in Fig. 6. For the curves of Figs. $5 \mathrm{a}$ and $6 \mathrm{a}$, the mean wind speed is $20 \mathrm{~m} / \mathrm{s}$, while the train speeds are 200 and $400 \mathrm{~km} / \mathrm{h}$,

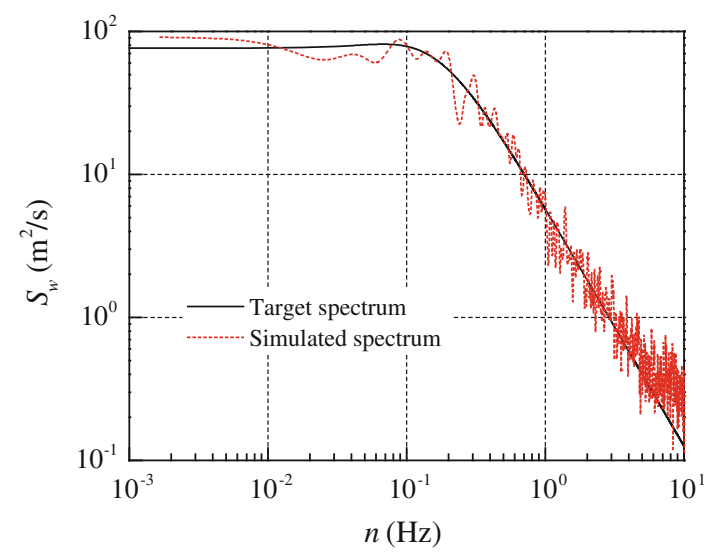

Fig. 4 Comparison of simulated spectrum and target spectrum

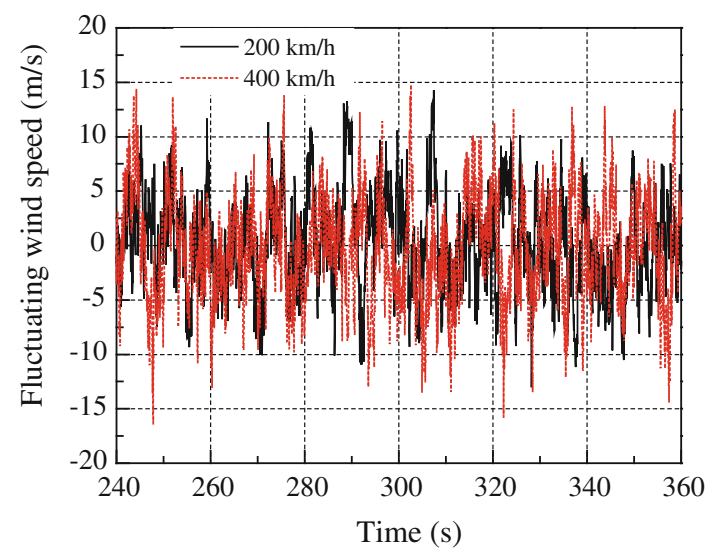

(a)

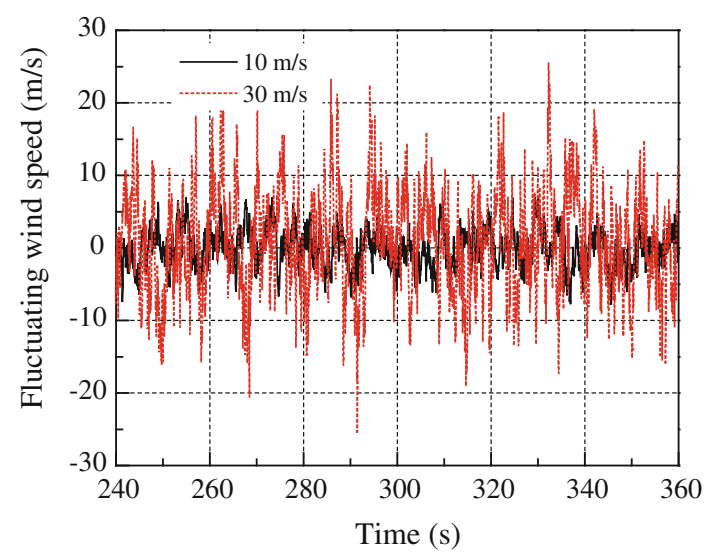

(b)

Fig. 5 Simulation of fluctuating wind speed at different train speeds (a) and different mean wind speeds (b)

respectively. For the curves of Figs. $5 \mathrm{~b}$ and $6 \mathrm{~b}$, the mean wind speeds are 10 and $30 \mathrm{~m} / \mathrm{s}$, respectively, while the train speed is $350 \mathrm{~km} / \mathrm{h}$.

From Figs. 5 and 6, we can find that when the train speed is fixed, the varying mean wind speeds have large 


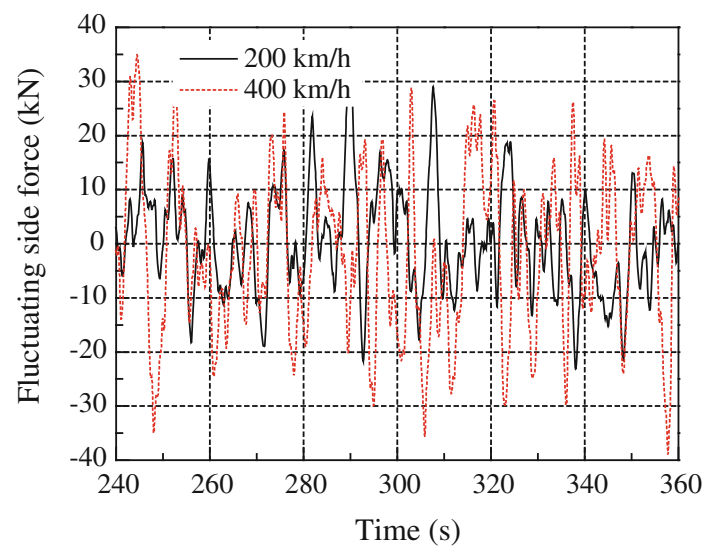

(a)

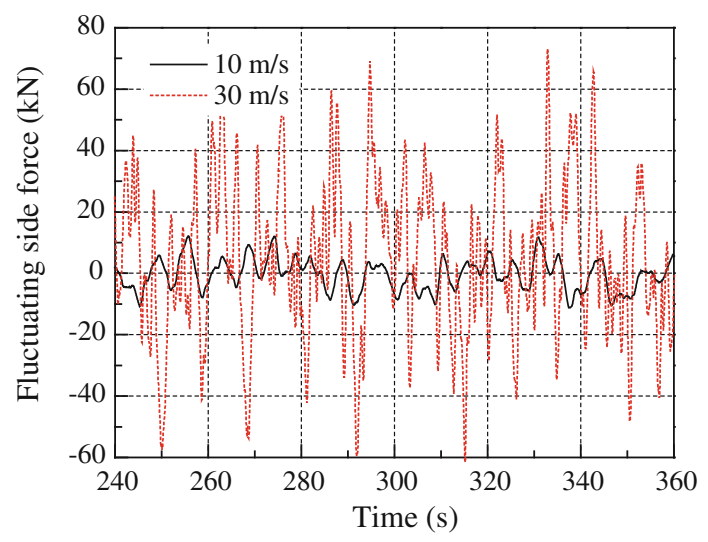

(b)

Fig. 6 Simulation of fluctuating side force at different train speeds (a) and different mean wind speeds (b)

impact on the fluctuating values of the wind speeds and aerodynamic side forces. However, when the wind speed is fixed, the varying train speeds have little impact on the fluctuating values of the wind speeds or aerodynamic side forces. These rules are applicable to other aerodynamic forces and moments.

\subsection{Statistical characteristics of aerodynamic loads}

Investigation of the statistical characteristics of aerodynamic loads needs a lot of wind samples. For each sample, the standard deviation and maximum value of aerodynamic loads are calculated. By averaging the values of multiple samples, more accurate standard deviation and maximum of aerodynamic loads can be obtained. The number of samples used in this study is 200 , from which we can obtain a more accurate standard deviation $\sigma_{F}$ and maximum $F_{\max }$.

The ratio of the standard deviation of the aerodynamic force (moment) to $0.5 \rho K \bar{u}^{2}$ at different mean yaw angles is shown in Fig. 7. We can see that the ratio of the standard

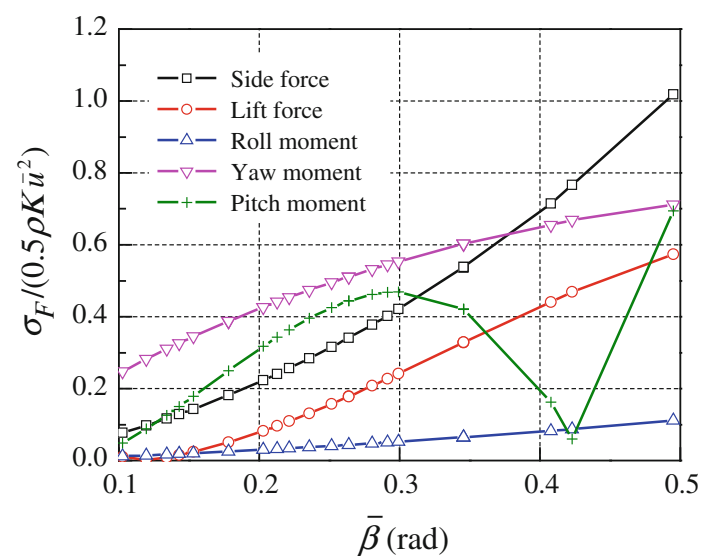

Fig. 7 Force (moment) standard deviation/ $0.5 \rho K \bar{u}^{2}$

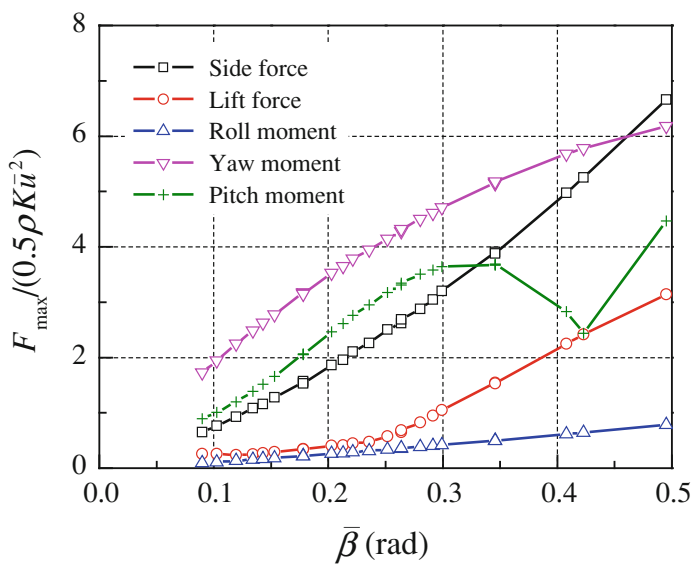

Fig. 8 Force (moment) maximum/0.5 $\rho K \bar{u}^{2}$

deviation of the aerodynamic force (moment) to $0.5 \rho K \bar{u}^{2}$ is the function of mean yaw angle. As the mean yaw angle increases, the ratios increase except that of the pitch moment, which varies in a more complex manner.

Figure 8 shows a plot of the ratio of the maximum value of the aerodynamic force (moment) to $0.5 \rho K \bar{u}^{2}$ at different mean yaw angles. The ratio of the maximum value of the aerodynamic force (moment) to $0.5 \rho K \bar{u}^{2}$ has the same variation as that of Fig. 7 .

The maximum aerodynamic forces (moments) can be expressed as [16]

$F_{\max }=\bar{F}+k_{F} \sigma_{F}$,

where $k_{F}$ is the peak factor of the aerodynamic force(moment), $\sigma_{F}$ can be obtained from the data of Fig. 7, $F_{\max }$ can be calculated from the data of Fig. 8 , and $\bar{F}$ can be derived from the Eq. (8).

Through the calculation, we find that the peak factor of lift force is $\sim 3.33$. The peak factors of the side force and roll moment are the same $(\sim 3.28)$. This is because the 
admittance functions of side force and roll moment are the same. The peak factors of pitch moment and yaw moment are the same $(\sim 3.77)$. This is because the pitch and yaw moment fluctuations follow the wind fluctuations.

\section{Conclusion}

In this paper, the aerodynamic characteristics of high-speed trains in fluctuating crosswinds are studied. It is revealed that the aerodynamic admittance function of the lift force is greater than that of the side force at the same mean yaw angle, and the admittance function of the aerodynamic force is moving to the higher part of the frequency as the mean yaw angles increase. When the train speed is fixed, the varying mean wind speeds have large impact on the fluctuating values of the wind speeds and aerodynamic loads; however, when the wind speed is fixed, the varying train speeds have little impact on the fluctuating values of the wind speeds or aerodynamic loads. The ratio of the standard deviation of the aerodynamic force (moment) to $0.5 \rho K \bar{u}^{2}$ is the function of the mean yaw angle. The ratio of the maximum value of the aerodynamic force (moment) to $0.5 \rho K \bar{u}^{2}$ at different mean yaw angles has the same variation as those of the standard deviation. The peak factors of aerodynamic loads are calculated in this paper. The peak factor of lift force is $\sim 3.33$. The peak factors of side force and roll moment are the same $(\sim 3.28)$, and the peak factor of pitch moment and yaw moment are also the same $(\sim 3.77)$.

Acknowledgments This research is supported by the 2013 Doctoral Innovation Funds of Southwest Jiaotong University and the Fundamental Research Funds for the Central Universities, the National Key Technology R\&D Program of China (2009BAG12A01-C09), and the High-Speed Railway Basic Research Fund Key Project (U1234208).

Open Access This article is distributed under the terms of the Creative Commons Attribution License which permits any use, distribution, and reproduction in any medium, provided the original author(s) and the source are credited.

\section{References}

1. Dierichs B, Sima M, Orellano A et al (2007) Crosswind stability of a high-speed train on a high embankment. J Rail Rapid Transit 221(2):205-225

2. Li T, Zhang JY, Zhang WH (2011) An Improved algorithm for fluid-structure interaction of high-speed trains under crosswind. J Modern Transp 19(2):75-81

3. Liu JL, Yu MG, Zhang JY et al (2011) Study on running safety of high-speed train under crosswind by large eddy simulation. J China Railway Soc. 33(4):13-21 (in Chinese)

4. Hoppmann U, Koening S, Tielkes T et al (2002) A short-term strong wind prediction model for railway application, design and verification. J Wind Eng Ind Aerodyn 90(10):1127-1134

5. Ge YM, Zhou SH, Li LA (2001) Coupled vibration of train and cable-stayed bridges with the effects of wind. J Southwest Jiaotong University 36(4):369-373 (in Chinese)

6. Wang SQ, Xia H, Guo WW et al (2012) Nonlinear coupling vibration analysis of wind load-train-long-span bridge system. J Beijing Jiaotong University 36(3):36-46 (in Chinese)

7. Cooper RK (1984) Atmospheric turbulence with respect to moving ground vehicles. J Wind Eng Ind Aerodyn 17(2):215-238

8. Baker CJ (2003) Some complex applications of the "wind loading chain". J Wind Eng Ind Aerodyn 91(12-15):1791-1811

9. Baker CJ, Jones J, Lopez-Calleja F et al (2004) Measurement of the cross wind forces on trains. J Wind Eng Ind Aerodyn 92(7-8):547-563

10. Sterling M, Baker CJ, Bouferrouk A et al (2009) An investigation of the aerodynamic admittances and aerodynamic weighting functions of trains. J Wind Eng Ind Aerodyn 97(11-12):512-522

11. Baker CJ (2010) The simulation of unsteady aerodynamic cross wind forces on trains. J Wind Eng Ind Aerodyn 98(2):88-99

12. Christian W, Carsten P (2008) Crosswind stability of high-speed trains: a stochastic approach. BBAA BI International Colloquium on: Bluff Bodies Aerodynamics \& Applications. Milano, 20-24:1-16

13. Standards Policy and Strategy Committee (2010) EN 14067-6: 2010 railway applications-aerodynamics-part 6: requirement and test procedures for cross wind assessment. CEN, Brussels

14. Sterling M, Baker CJ, Bouferrouk A et al (2009) An investigation of the aerodynamic admittances and aerodynamic admittances and aerodynamic weighting functions of trains. J Wind Eng Ind Aerodyn 97(11-12):512-522

15. Yu MG, Zhang JY, Zhang WH (2011) Wind-induced security of high-speed trains on the ground. J Southwest Jiaotong University 46(6):989-995 (in Chinese)

16. Dyrbye C, Hansen SO (1996) Wind loads on structures. Wiley, New York 